\title{
Defining and Measuring Trophic Role Similarity in Food Webs Using Regular Equivalence
}

\author{
Joseph J. Luczkovich* ${ }^{*}$, Stephen P. Borgatti \\ and Martin G. Everett $\|$
}

\begin{abstract}
$\dagger$ Department of Biology, Institute for Coastal and Marine Resources, East Carolina University, Greenville, NC 27858, U.S.A. \$Organization Studies Department, Carroll Graduate School of Management, Boston College, Chestnut Hill, MA 02467, U.S.A. §Institute for Coastal and Marine Resources, Department of Sociology, East Carolina University, Greenville, NC 27858, U.S.A. and " School of Computing and Mathematical Sciences, University of Greenwich, London SE10-9LS, U.K.
\end{abstract}

(Received on 8 October 2001, Accepted in revised form on 13 August 2002)

\begin{abstract}
We present a graph theoretic model of analysing food web structure called regular equivalence. Regular equivalence is a method for partitioning the species in a food web into "isotrophic classes" that play the same structural roles, even if they are not directly consuming the same prey or if they do not share the same predators. We contrast regular equivalence models, in which two species are members of the same trophic group if they have trophic links to the same set of other trophic groups, with structural equivalence models, in which species are equivalent if they are connected to the exact same other species. Here, the regular equivalence approach is applied to two published food webs: (1) a topological web (Malaysian pitcher plant insect food web) and (2) a carbon-flow web (St. Marks, Florida seagrass ecosystem food web). Regular equivalence produced a more satisfactory set of classes than did the structural approach, grouping basal taxa with other basal taxa and not with top predators. Regular equivalence models provide a way to mathematically formalize trophic position, trophic group and trophic niche. These models are part of a family of models that includes structural models used extensively by ecologists now. Regular equivalence models uncover similarities in trophic roles at a higher level of organization than do the structural models. The approach outlined is useful for measuring the trophic roles of species in food web models, measuring similarity in trophic relations of two or more species, comparing food webs over time and across geographic regions, and aggregating taxa into trophic groups that reduce the complexity of ecosystem feeding relations without obscuring network relationships. In addition, we hope the approach will prove useful in predicting the outcome of predator-prey interactions in experimental studies.
\end{abstract}

(C) 2003 Elsevier Science Ltd. All rights reserved.

*Corresponding author. Tel.: +1-252-328-1847; fax: $+1-252-328-4178$.

E-mail address: luczkovichj@mail.ecu.edu (J.J. Luczkovich).

\section{Introduction}

The notion of trophic role has been central to the development of the concept of ecological niche and food web research. Although the idea of trophic role of an organism is nearly as old as ecology itself, the concept has never truly been 
formally defined. Elton (1927) first defined the niche as the fundamental role of an organism in its community - largely in its relation to food and to enemies. In defining a species' role in the community, Elton drew an analogy with social roles in human communities. Since that early definition, numerous authors have discussed ideas related to trophic role, defining and using different terms such as trophic level (Lindemann, 1942), trophic niche (Hutchinson, 1958), ecological guild (Root, 1967), and trophospecies (Yodzis, 1988). Although each of these terms in some way defines a species' trophic role in an ecosystem, each concept differs slightly, and none is completely satisfactory in capturing the total feeding relations of a species in a food web (Cousins, 1985, 1987; Perrson et al., 1996). Here, we formally define the trophic role of a species using a concept drawn from social role analysis called regular equivalence, and present examples using this approach to measuring similarity in the trophic role of species in food webs.

Recently, some new approaches to the definition and measurement of the trophic role of a species in a food web have been proposed, in which both predator and prey relations are considered (Goldwasser \& Roughgarden, 1993). Yodzis \& Winemiller (1999) used Jaccard's (1900) similarity coefficient to operationally define trophospecies, first computing predator relations, then prey relations, and finally both sets of relations at the same time. Given the matrix of species-by-species Jaccard similarity coefficients, they used clustering algorithms to identify classes of species of fishes in an aquatic food web in a Venezuelan floodplain. They considered two separate sets of a species' links to other species (using both topological and energy flow links in separate analyses), first computing the similarity of predator links, then computing the similarity of prey links, then multiplying the similarity measures - what they called the multiplicative similarity. They also analysed trophic similarity by ignoring the distinction between predator and prey, simultaneously computing similarity in the dual role each species played as a consumer and a resource - what they called the additive similarity. They concluded that the additive similarity measure provided the best measure of trophic similarity based on predator and prey relations. The Yodzis-Winemiller approach is an important advance because it formalizes a fundamental concept (providing all the associated benefits of precision and measurability), and because it incorporates connections up and down the food web. However, it also retains an important weakness in the classical conceptualization of trophic role: it fails to detect the equivalence of roles of species that do not share the same predators and prey, but that may be similar in their trophic position.

As an example, consider two species of insect that serve as prey for two different species of congeneric birds, and which consume very similar, congeneric species of plants. In the Yodzis-Winemiller approach, these two insect species would be placed in separate trophic groups because they share no predators and no prey. Yet this measure misses the similarity at a higher level of trophic organization: both species have a similar trophic position within the food web. Both insects are functional herbivores, and they are eaten by very similar species, but there is no way for the Yodzis-Winemiller approach to detect that these two have any more in common than any random pair of species that also share no prey or predators. One solution would be to modify the Yodzis-Winemiller approach to measure similarity not at the species level but at a more aggregate level. For example, in computing the shared prey coefficient for each insect species, we could hypothetically count not how many species they have in common, but how many genera they eat in common. Similarly, in computing the shared predator coefficient, we could count the number of shared bird genera they have in common. The obvious problem with this is that we do not know that the genus is the right level of aggregation, nor do we have any basis for choosing phylogenetic affiliation as the basis for grouping. However, the general idea is right: to evaluate the trophic similarity of two species, we want to know whether they have similar trophic ties to the same types of species, but not necessarily the same species. The question is how to define these types. Ideally, we would use trophospecies - groups of species that are trophically equivalent. But this would seem to require knowing the answer in order to 
construct the answer. We are trying to group species into trophically equivalent sets, yet to do this for any given pair of species, we would like to know which trophic equivalence set all of the other species belong to, which is circular. Surprisingly, there is a solution, which is recursive in nature; and, it is drawn from the social sciences, where the concept of social role of individuals within social systems has been extensively analysed.

The social sciences have paid much attention to the importance of structure and position in the study of social roles. Social scientists have devoted a considerable amount of effort to formalizing the notion of role in the context of a system of social relations (Nadel, 1957; Merton, 1959; Homans, 1961; Goodenough, 1969; Mayhew, 1980). For example, in the system of social relations in a hospital, what defines a person as playing the role of doctor is the characteristic set of relations they have with persons who are playing related roles such as patients, nurses, medical record keepers, pharmaceutical sales people and receptionists. Early social network research modeled kinship systems using algebraic approaches (White, 1963; Boyd, 1969), followed by Lorrain \& White's (1971) "structural equivalence" concept that formally defined roles and reduced the complexity of a network of relations to a set of simplified role structures. In this structural equivalence model, two individuals are seen as occupying the same position to the extent they have the same kinds of social ties to the same third parties. This work in turn paved the way for the development of a more general concept known as regular equivalence (White \& Reitz, 1983), in which two individuals are seen as playing the same role to the extent they have similar ties to analogous individuals (i.e. those playing corresponding roles). Differences between structural and regular equivalence approaches were discussed by Borgatti \& Everett (1989), Everett \& Borgatti (1991) and Everett \& Borgatti (1994).

As an extension of Elton's (1927) "role in relation to food and enemies" paradigm, we will now apply the theory of social role analysis and the concept of regular equivalence to ecological food webs and formalize the measurements of trophic role similarity in detail. We will apply the regular equivalence approach to the analysis of trophic role structure in two food webs, one a binary or topological network and the other a carbon-flow network. We will also compare performance of the regular equivalence approach with the structural method proposed recently (Yodzis \& Winemiller, 1999) in creating trophic aggregations. Finally, we will present an image graph of each food web, based on the regular equivalence approach, which provides a reduced-complexity view of the entire food web.

\section{Theory of Role Analysis}

\subsection{GRAPH-THEORETIC TERMINOLOGY}

We represent community food web data as a directed graph, or digraph, $G(V, E)$, which consists of a set of nodes $V$ (also known as vertices) representing species or compartments, and a set of directed ties (also known as edges or arcs) $E$ which represent predation, parasitism or any other trophic relationship. The notation $(a, b) \in E$ indicates the presence of a tie from $a$ to $b$. Similarly, $(b, a) \in E$, indicates a tie from $b$ to $a$. Optionally, we can also define a real-valued function $F$ on $E$ which assigns a value (such as a quantity of flow) to each tie, so that $f(u, v)$ $=0.27$ would indicate a flow of 0.27 units from $u$ to $v$. For simplicity of exposition, we shall usually assume here that ties are simply present or absent rather than valued. However, the mathematics generalizes straightforwardly to valued data, and we will use valued energy flow data in our empirical examples.

The set of nodes adjacent to a given node $v$ is called the neighborhood of $v$ and denoted $N(v)$. In a directed graph, a node's neighborhood consists of two parts: an out-neighborhood, defined as the set $N^{o}(v)=\{p \mid(v, p) \in E\}$, and an in-neighborhood, defined as the set $N^{i}(v)=$ $\{q \mid(q, v) \in E\}$. In the case of food web predation data, the out-neighborhood $N^{o}(u)$ is the set of species that are predators of species $u$, and the in-neighborhood $N^{i}(u)$ is the set of species that are prey of species $u$.

An equivalence relation defined on a set of nodes is a binary relation that is reflexive, symmetric and transitive. An example of an equivalence relation is the relation $R$ induced by a partition of nodes into mutually exclusive 
classes so that two nodes are related by $R$ if and only if they belong to the same class. The notation $(u, v) \in R$ is used to indicate that node $u$ is equivalent (i.e. in the same class as) to node $v$. Alternatively, we can write $u \equiv v$ to indicate the equivalence of the two nodes. The equivalence class of a node $u$ is denoted $C(u)$. Below, we define two particular equivalence relations: structural equivalence, in which nodes are equivalent if they are connected to the same other nodes; and regular colorations, in which equivalent nodes are connected to equivalent (but not necessarily the same) nodes.

\subsection{STRUCTURAL EQUIVALENCE}

An equivalence relation $R$ is called structural (Lorrain \& White, 1971; Everett \& Borgatti, 1991) if, for all nodes $u$ and $v,(u, v) \in R$ if and only if $N^{o}(u)=N^{o}(v)$ and $N^{i}(u)=N^{i}(v)$. In other words, a structural equivalence - also known as a structural coloration (Everett \& Borgatti, 1991) - equates nodes that have incoming and outgoing ties to exactly the same others. Figure 1(a) illustrates a structural equivalence by painting equivalent nodes the same color. In contrast, the equivalence depicted in Fig. 1(b) is not structural because nodes 5 and 6 are colored the same, yet node 9 has an outgoing tie to node 5 , and node 6 does not. In addition, node 5 has an outgoing tie to node 4 , and node 6 does not. In structural equivalence, nodes 5 and 6 could not be equivalent. For valued data, such as energy flows, we would require that fully equivalent nodes have equally valued ties to the same others.

As defined above, structural equivalence is an idealized mathematical concept in which nodes are either equivalent or they are not. When using the concept to analyse empirical data, we use structural equivalence algorithms (Borgatti et al., 1999) that measure the extent of structural equivalence between every pair of nodes. When the appropriate measure of pattern similarity is chosen (such as the Jaccard coefficient), the latter approach is almost identical to recent approaches used by ecologists for identifying trophospecies (Yodzis \& Winemiller, 1999).

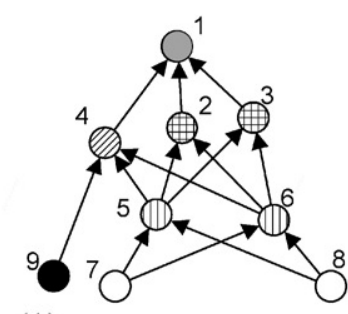

(A)

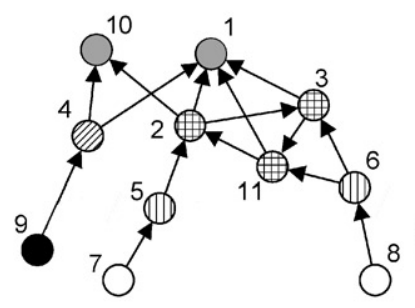

(C)

(D)

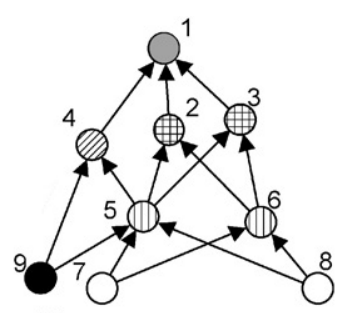

(B)
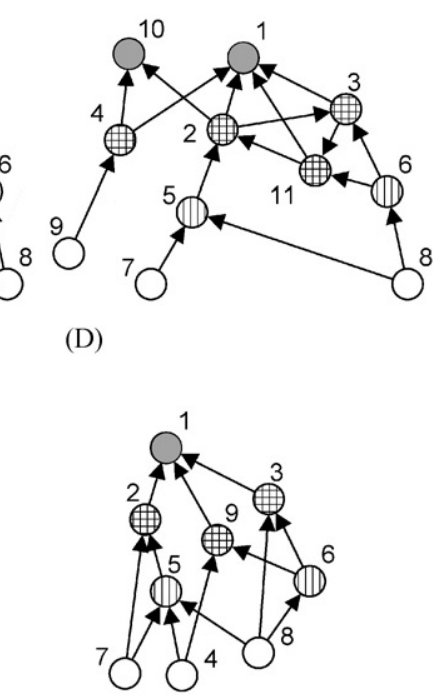

FIG. 1. (a) A structural coloration (left), (b) a nonstructural coloration (right), (c) a regular coloration, (d) a non-regular coloration, (e) disconnected graphs representing different webs. Color codes: solid circles, black; open circles, white; angled hatching, green; vertical hatching, red; cross hatching, blue; stipled, yellow.

\subsection{REGULAR EQUIVALENCE}

Structural equivalences are members of a family of equivalence relations called regular equivalences or regular colorations (White \& Reitz, 1983; Borgatti \& Everett, 1989; Everett \& Borgatti, 1991). An equivalence relation $R$ of a digraph $G(V, E)$ is regular if, for all nodes $u, v \in$ $V, u \equiv v$ implies that if there exists a tie $(u, y) \in E$, then there exists a node $z$ such that $(v, z) \in \mathrm{E}$ and $y \equiv z$, and if there is a tie $(p, u) \in E$, then there exists a node $q$ such that $(q, v) \in E$ and $p \equiv q$. In other words, in a regular equivalence, if nodes $u$ and $v$ are equivalent, then if one has a tie to a third party, then the other one has a corresponding tie to an equivalent third party (but not necessarily the same one). Applied to food webs, this means that regularly equivalent species prey on equivalent species and are preyed upon by equivalent species. It should be noted that a 
graph may have more than one regular equivalence. Since our goal is usually to seek the simplest model, our discussion will center on the maximal non-trivial regular equivalence (i.e. the one with the fewest equivalence classes). Consequently, when we write "regular equivalence" it is understood that this means the maximal regular equivalence unless otherwise stated.

An example of a regular equivalence is shown in Fig. 1(c), again using same color and fill to indicate equivalence. To determine whether it is regular, we check each pair of nodes that is colored the same against the definition. For example, nodes 2 and 3 are colored the same, indicating they are equivalent. According to the definition, their out-neighborhoods (and their inneighborhoods) must contain equivalent nodes (i.e. the same colors). Node 2 has incoming ties from cross-hatched nodes and vertical-lined nodes and no other colors. Node 3 is the same. Node 2 has outgoing ties to stippled nodes and cross-hatched nodes. Node 3 is again the same (recall that the definition says nothing about the number of nodes of each type that a node may be connected to). Hence this pair satisfies the definition. If all pairs do, the equivalence is regular. In contrast, Fig. 1(d) gives an example of a non-regular equivalence. One reason it is not regular is that node 4 is colored the same as node 2 , yet node 4 has no tie to a vertical-lined node, while node 2 does.

It is useful to note three desirable features of regular equivalence. The first is that cycles, such as the one connecting nodes 2, 3 and 11 in Fig. 1(c), pose no difficulty. The second is that omnivory also provides no particular problem, as nodes 10 and 1 feed at the same two levels of the food chain (and are equivalent) whereas nodes 4 and 2 feed at different levels (and are not equivalent). The third is that two species can be regularly equivalent without having any prey or predators in common, as is the case with nodes 5 and 6 . Thus, regular equivalence captures the idea that species can occupy analogous positions in the food web, without the limitation found in the Yodzis-Winemiller approach that requires species assigned to the same class to interact trophically with the very same species. An implication of the last feature is that regular equivalence can be used to class together species

that are members of different food webs, yet occupy analogous positions. An example is given in Fig. 1(e) in which nodes 12 and 2 are regularly equivalent, yet obviously belong to separate webs. In this way, regular equivalence may be used to improve upon the practice of comparing webs by counting numbers of species by basal-, intermediate- and top-level species (Cohen \& Newman, 1985).

\subsection{IMAGE GRAPHS}

The image graph induced by an equivalence relation $\mathrm{R}$ on $G$ is defined as the digraph $G^{\prime}\left(C(V), E^{\prime}\right)$ in which the nodes are the equivalence classes of $R$, and two nodes (i.e. classes) $x$ and $y$ are adjacent in $G^{\prime}$ if there exist adjacent nodes $u$ and $v$ in $G$ such that $C(u)=x$ and $C(v)$ $=y$ [recall that $C(u)$ denotes the equivalence class of node $u$ ]. In this sense, an equivalence relation induces a mapping, known as a graph homomorphism, from the original network to a reduced model of the network. Figure 2(a) shows the image graph for the structural equivalence in Fig. 1(a). It should be noted that in a structural equivalence, the image graph is a complete description of the entire system of relations,

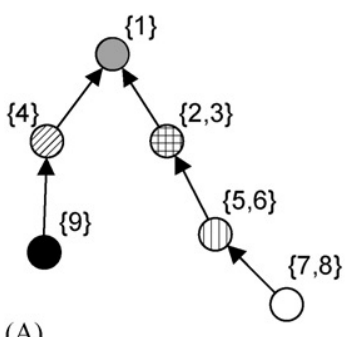

(A)

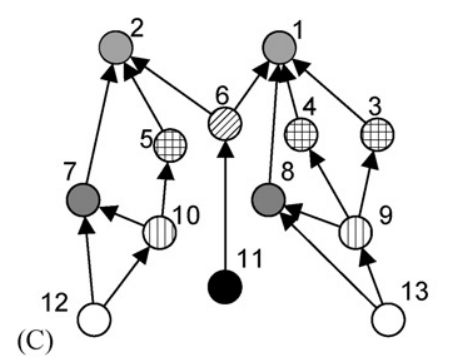

FIG. 2. (a) Image graph for the structural coloration in Fig. 1(a); (b) image graph for the regular coloration in Fig. 1(c); (c) a graph of a hypothetical food web showing the regular equivalence of feeding relations. Directed ties are depicted by arrows that show the predator's consumption of a prey. Color codes are the same as in Fig. 1. 
without loss of relational information. If two colors blue and red are mutually connected in the image graph, then, in the original graph, every blue node has a connection to every red node, and every red node has an incoming tie from every blue node.

The image graph of a regular equivalence also provides a reduced model of the network(s) as a whole. Unlike structural equivalence, however, with regular equivalence there is some loss of relational information, although the extent and type of loss are strictly controlled. Figure 2(b) gives the image graph associated with the regular equivalence in Fig. 1(e). Given the rules of a regular equivalence, we are guaranteed that if, in the image graph, there is a tie from a verticallined node to a cross-hatched node, then in the original graph, every cross-hatched node has an incoming tie from at least one vertical-lined node, and every vertical-lined node has an outgoing tie to at least one cross-hatched node. Thus, the image graph from a regular equivalence provides a simplified graph of the pattern of connections among individual species; it is a disciplined way of representing complex food web structure in which some detailed information is lost, but the fundamental pattern or structure of ties is preserved.

A look at Fig. 2(c) suggests that in addition to capturing the notion of species trophic role, regular equivalence has some relationship to the notion of trophic position. Node 3, which is cross-hatched, is two links above a primary producer, and one link below a top predator. All the other cross-hatched nodes are positioned the same. Note that nodes 7 and 8 are very much like the cross-hatched nodes: they have an outgoing tie to the top predator, and an incoming tie from a vertical-lined prey. But unlike the crosshatched nodes, they also have a tie directly to the primary producers (white nodes), i.e. they are classic omnivores. As a result, they are placed in a different class, which corresponds to a different trophic position. Similarly, node 6 , which also is prey for the top predators, is in a different class from the vertical-lined nodes, the cross-hatched nodes and the gray nodes because it feeds directly and exclusively on a primary producer. Note that this means that node 11 , the primary producer connected to node 6 , cannot be in the same class as the other producers 12 and 13. In this sense, regular equivalence makes finer distinctions than do most other measures of trophic position, because the producers will be grouped into separate classes with differing relations to the herbivore and omnivore classes. Consequently, we can think of regular equivalence as defining classes of isotrophic species, rather than trophic levels of species.

The relationship between regular equivalence and trophic position is clearest when the food web contains no cycles. In such webs, two species that are colored the same are equally distant from corresponding top predators and producers. For example, if $a \equiv b$, then if $a$ is positioned two links above a given producer species $c$, then $b$ is also two links above either $c$ or some other producer $d$ that is regularly equivalent to $c$. Thus, under such circumstances, nodes in the same class are necessarily at the same trophic position. In food webs with cycles, the correspondence noted above can break down. However, there is a simple solution. In graph theory, the length of the shortest path between two nodes is known as the geodesic distance between them. By calculating the geodesic distance between all pairs of nodes, we can construct a node-by-node geodesic distance matrix. Applying regular equivalence to this matrix instead of the raw adjacency matrix generates a regular equivalence that necessarily preserves trophic levels, as two nodes that are colored the same will be the same geodesic distance from equivalent others. This approach can also be applied to flow data by applying regular equivalence simultaneously to both the flow matrix and the geodesic distance matrix, but the details of this technique are beyond the scope of this paper.

While regular equivalence classes are homogeneous with respect to trophic position, as explained above, they produce classes that differ from the groupings that result using the classic definition of trophic level. One reason is that trophic-level concept considers only distance from the producers, and does not include both downward and upward trophic paths. For example, if two nodes $a$ and $b$ are both two links above the producer, then they are not always regularly equivalent, because they may differ in the number of predator links above 
them in the web. In addition, some approaches, such as Adams et al. (1983), are sensitive to the relative number of prey at different levels, which is not the case with regular equivalence in general, although it is true of a subset of regular colorations known as exact regular colorations (Everett \& Borgatti, 1996). For example, in Fig. 2(c), nodes 1 and 2 are placed at the same level by a regular coloration, even though node 1 preys on two cross-hatched species while node 2 preys on only one.

A distinctive feature of the definition of regular equivalence is its recursive or implicit quality. That is, to determine whether two nodes are equivalent, one needs to know the colors of all the other nodes, but to determine their colors one needs to know the colors of all nodes including the two nodes one started with, and so on. This may appear to be computationally intractable, but in fact is not. There are several efficient algorithms available for regular equivalence (Borgatti et al., 1999), which vary in the type of output they produce (e.g. pairwise coefficients giving the degree of equivalence vs. approximate discrete classes), type of data they can handle (e.g. valued or binary), and other variables.

\section{Methods}

\subsection{EMPIRICAL APPLICATIONS}

In the last section we laid out the mathematical and computational underpinnings of the regular equivalence model. Now we illustrate the empirical application of these concepts using two empirical food web datasets, namely the Malaysian pitcher plant insect web (Beaver, 1985) as reproduced and discussed in Stiling (2002), and the St. Marks, Florida seagrass food web (Baird et al., 1998; Christian \& Luczkovich, 1999; Luczkovich et al., in press). The insect dataset is a topological web consisting of predation links, while the St. Marks, Florida seagrass ecosystem is a dynamic web, with data consisting of estimated carbon flows between compartments.

\subsection{MALAYSIAN PITCHER PLANT WEB}

This is one of several food webs that have been described by Beaver (1985), involving insects that are attracted to a Malaysian pitcher plant and become drowned or are preyed upon by other insects that live in the plant. In a sense, it is merely a sub-web of a larger food web, because the insects are consuming energy elsewhere and bringing it to the pitcher plant. Thus, the role of "producers" is filled by the drowned insects and live insects (ants) that visit the pitcher plant, which are really consumers in a larger food web. In the original presentation (Beaver, 1985), some taxa were grouped into "trophic types", which in fact were structurally equivalent, because they had the exact same links to other taxa in the food web. Stiling (2002) disaggregated these taxa, and we did as well, so as to start with a completely disaggregated food web at the species level (Fig. 3).

\subsection{ST. MARKS, FLORIDA SEAGRASS CARBON FLOW WEB}

The food web data were obtained from direct sampling and literature surveys of the St. Marks seagrass ecosystem (Baird et al., 1998; Christian \& Luczkovich, 1999, Luczkovich et al., in press).

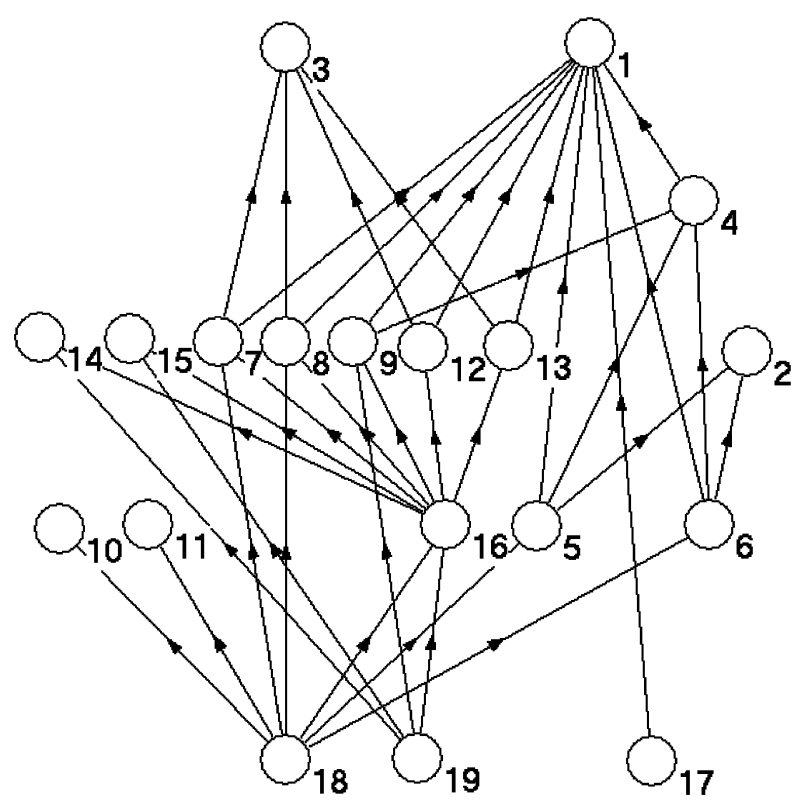

FIG. 3. A simple food web diagram of the insects in the pitcher plant Nepenthes albomarginata in West Malaysia [modified from the diagram presented by Stiling (2001), based on orginal data from Beaver, 1985]. Appendix A lists the species' numerical codes for each node. Predators are positioned higher than their prey, and lines represent trophic linkages. 
In order to obtain a species list for the food web, collections of producers and various consumers were conducted along the study area transects (for more detailed methods see Baird et al., 1998; Christian \& Luczkovich, 1999; Luczkovich et al. in press). In order to determine the structure of the diet matrix required for the network food web model, diet data were summarized based on the existing literature on seagrass invertebrates and birds, and stomachs were examined directly for fishes in the study areas. A 51-compartment diet matrix for this study was prepared as a carbon-flow matrix taken directly from the network model based on the approach described by Ulanowicz (1986).

\subsection{STATISTICAL ANALYSIS}

To analyse the data, we used the REGE algorithm implemented in the UCINET 5.8 network analysis software package (Borgatti et al., 1999). The algorithm takes any real-valued $N \times N$ (species-by-species) matrix $X$ as input, and returns a species-by-species matrix $R$ of coefficients (ranging from 0 to 1 ) which records, for each pair of species, the extent of (maximal) regular equivalence. The essence of the algorithm is as follows:

(0) Set $r_{i j}=1$ for all $\mathrm{i}$ and $\mathrm{j}$ (i.e. let all species be $100 \%$ equivalent to start).

(1) For each species $i$ and $j$,

(1A) For each species $k$ eaten by $i$, find species $m$ eaten by $j$ that is most equivalent to $k$ and which is eaten in the most similar proportion as $k$ is eaten by $i$, in other words which maximizes the quantity $z_{k}=r_{k m} \operatorname{Min}\left(x_{i k}, x_{j m}\right) / \operatorname{Max}\left(x_{i k}, x_{j m}\right)$.

(1B) For each $k$ which eats $i$, find species $m$ that eats $j$ that is most equivalent to $k$ and which eats $j$ in the most similar proportion as $k$ eats $i$, in other words which maximizes the quantity $y_{k}=r_{k m} \operatorname{Min}\left(x_{k i}, x_{m j}\right) / \operatorname{Max}\left(x_{k i}, x_{m j}\right)$.

(1C) Set $r_{i j}$ and $r_{j i}=\Sigma z_{k}+\Sigma y_{k}$.

(2) Repeat Step 1 until no more changes in $r_{i j}$ or maximum iterations exceeded. The maximum iterations $=N$ species or compartments.

The resulting coefficients $r_{i j}$ have ordinal properties. Details of the algorithm are discussed in Borgatti \& Everett (1993). For comparison, we also calculate the additive similarity measure of Yodzis \& Winemiller (1999) for the Malaysian pitcher plant insect food web, which is Jaccard's similarity measure applied to all trophic links, whether predator or prey of a species. To visualize patterns of similarities emerging from both the REGE procedure and the YodzisWinemiller procedure, the similarity matrices were submitted to a non-metric multi-dimensional scaling (MDS) procedure in order to represent similarities as distances in two-dimensional space (UCINET 5, Borgatti et al., 1999). The coordinates from the MDS procedure were then used as input to the Pajek (Batagelj \& Mrvar, 1999) network drawing software.

In addition, in order to simplify interpretation of the results, a hierarchical clustering of the output matrix $R$ from the REGE algorithm was also performed, yielding a dendrogram and set of nested partitions. Because it is appropriate for ordinal data, we used Johnson's hierarchical clustering (Johnson, 1967). For display purposes, one partition within the hierarchical clustering was selected to classify compartments. By definition, all partitions within a Johnson's hierarchical clustering are equally valid, representing different levels of resolution rather than alternative theories (Borgatti et al., 1990). The particular choice of partition was based on a series of regressions designed to measure cluster adequacy. Since an ideal clustering of the $R$ matrix would locate the largest values of $R$ within clusters and the smallest values of $R$ between clusters, we can measure the extent to which a given clustering is optimal via an analysis of variance in which the cases are pairs of nodes, the dependent variable is the REGE coefficient for each pair, and the independent variable is a dummy variable coded 1 if the pair are in the same cluster and 0 if they are in different clusters. The resulting $R^{2}$ (or eta-square as it is called in the ANOVA context) can be interpreted as a measure of cluster adequacy. By plotting $R^{2}$ against the number of clusters, we obtain a scree plot, which can be examined for inflection points. A clustering with $k$ classes is chosen if it provides a sizeable increase in $R^{2}$ over the next simplest clustering (i.e. with $k-1$ clusters), yet explains nearly as much variance as the next most complicated clustering $(k+1$ 
clusters). Of course, the researcher is free to choose a cluster solution with higher resolution if the $R^{2}$ is not substantially different.

\section{Results}

\subsection{MALAYSIAN PITCHER PLANT INSECT FOOD WEB}

The 19 taxa in the topological food web (Fig. 3) collected by Beaver (1985) and discussed in Stiling (2002) were analysed via the REGE algorithm, generating the matrix of coefficients (available upon request from the authors and published on-line at http://drjoe.biology.ecu. edu/regefoodweb). Appendix A lists the names and numerical identification codes for the compartments in Beaver (1985) that are discussed here. It is clear that the REGE algorithm does a very good job of detecting isotrophic species in the Malaysian pitcher plant insect food web. Based on the increase in $R^{2}$ of the regression analysis at four clusters and the rapid drop after five clusters, as can be seen in the cluster adequacy scree diagram (Fig. 4), we identified five isotrophic classes (with one singleton class), which are identified by colors in the cluster dendrogram (Fig. 5).

Figure 6(a) shows a two-dimensional multidimensional scaling (MDS) of the REGE coefficients for this web, with the color classes from the cluster analysis used to code the classes. This MDS displays the food web structure and

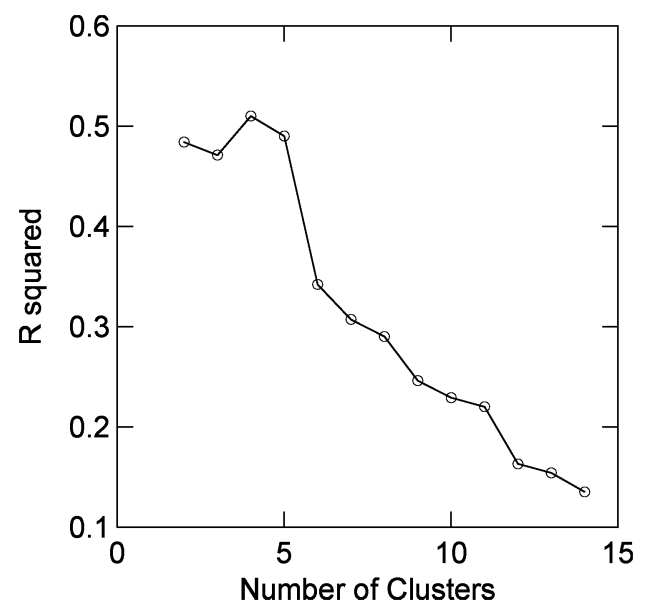

FIG. 4. A screen plot of the $R^{2}$ as it varies with number of clusters from the average linkage cluster analysis using the REGE coefficients derived from the Malaysian pitcher plant insect food web. relative position in the network of the basal, intermediate and top consumers. One group of basal species (brown) included the drowned insects (18) and organic debris (19). Another basal group (singleton class) includes the live insects (white) (17). Both basal groups have no incoming ties - they function as the energy source or "producers" in this food web. The REGE algorithm separates basal species into two classes (brown and white), based on the relations of those nodes to the various color classes of their consumers: whereas brown class members are preyed upon by members of the green and red classes, the white class members are only preyed upon by members of the yellow class. The intermediate group (green) includes the bacteria and protozoa (16), aquatic larvae of dipteran insects [Megaselia sp. (5), Endonepenthia schuitemakeri (6), Triperoides tenax (7), Triperoides bambusa (8), Dasychelea nepenthicola (9), Culex curtipalpis (12), Culex lucaris (13) and Lestodiplosis sp. (4)]. These intermediate aquatic insect larvae (green) are positioned midway from top to bottom in the MDS plot, which indicates that they occupy a position midway between food sources and sinks. There were two top

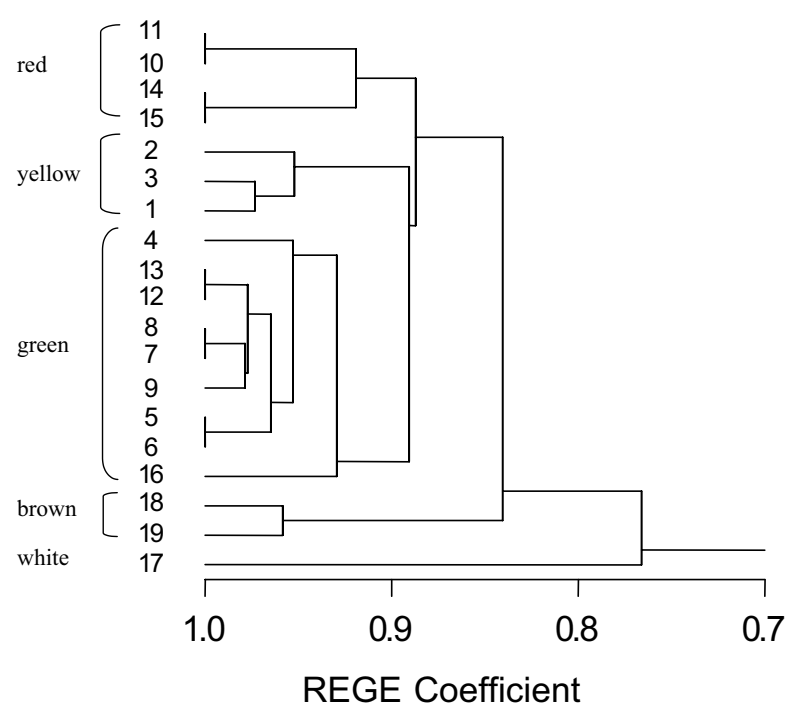

FIG. 5. A single-linkage cluster analysis dendrogram of the Malaysian pitcher plant insect food web using the REGE coefficient matrix as a similarity measure (Taxa codes listed in Appendix A). Colors were used to indentify class membership based on the $R^{2}$ regression analysis (see text). 
consumer groups: the red group included scavenging dipterans Nepenthosyrphus sp. (10), Pierretia urceola (11), and two species of Anotidae (14 and 15); whereas the predatory insects Misumenops nepenthicola (1), encyrtids (Trachinaephagus) (2) and Toxorhynchies klossi (3) comprised the yellow group. Both the yellow and red groups are sinks for energy in this web (they have no outgoing ties).

Five pairs of species were exactly regularly equivalent, with coefficients of 1.0 (5 and $6 ; 7$ and $8 ; 10$ and $11 ; 12$ and 13; and 14 and 15). These nodes are shown as slightly displaced from one another in Fig. 6(a) to prevent them from completely obscuring one another. These pairs of nodes have the same ties to the same classes of species, but not necessarily the same species. In this example, each pair is tied to the exact same species, considering both predators and prey, so they are both regularly and structurally equivalent.

The complexity of the feeding relations in this food web network at the level of species can now be simplified from the original 19 nodes into five color classes. After pooling nodes in the same color class into a single node and redisplaying the network of these pooled nodes, we created a regular-equivalence image graph. The image graph of the pitcher plant food web shows the network structure in simplified form [Fig. 6(b)].

For comparative purposes, we also have analysed the same food web data using Yodzis \& Winemiller's (1999) preferred similarity measure, the additive trophic similarity measure, which is a measure of structural equivalence (Fig. 7 - similarity data available from the authors or at http://drjoe.biology.ecu.edu/regefoodweb). The two types of similarity measures, structural (additive similarities) and the regular (REGE coefficients) equivalence, produced two very different views of similarities among the species (compare Figs 5 and 7). Whereas the hierarchical clustering based on REGE coefficients grouped the basal producers into the same class (drowned insects 18, organic debris 19) at an early step, in the additive similarity clustering they are not joined until the next to the last agglomeration step (Fig. 7). According to the additive similarity measure, the node for organic debris (19) is more similar to the top predator $M$. nepenthicola (1) than it is to drowned insects
(18). The additive similarity measure produces a non-intuitive grouping of nodes in the food web. We can visualize the structural equivalence of the network if we compute the MDS coordinates in two dimensions using the additive similarity measure and display these with the network trophic links [Fig. 6(c)], as we did above with the REGE coefficients [refer to Fig. 6(a) for comparison]. The food web depicted with nodes positioned according to the MDS coordinates obtained from the additive similarity measure does not describe the flow of energy or materials from sources to sinks, as it did when REGE coefficients were used. Indeed, the basal nodes (17-19) are not particularly close to one another, and they cannot be arranged in such a way that they all lie near one end of the graph. The top predators nodes (1-3) are close to one another and on one side of the plot, but are also in close proximity to one of the basal nodes (18). This is a characteristic of the additive measure, which ignores the direction of the ties. In fact, the arrows representing energy flow among nodes in Fig. 6(c) do not all point in the same general direction, rather they cross back over one another, so there is no approximation of trophic position that can be uncovered in their arrangement by MDS. A few pairs of nodes appear to group together: these are species with identical

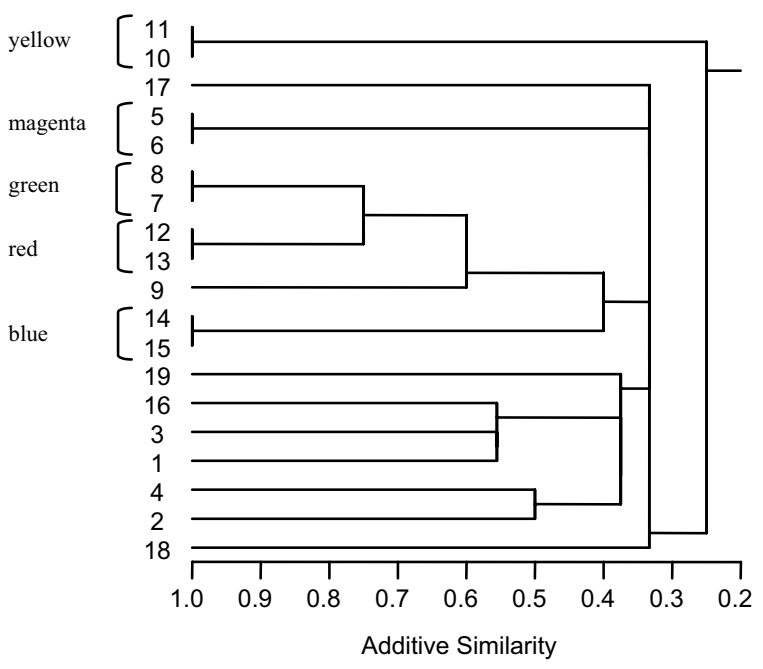

FIG. 7. An average-linkage cluster diagram of the Malaysian pitcher plant insect food web using the additive similarity measure of Yodzis \& Winemiller (1999). 
predator and prey relations, which thus have additive similarities of 1.0 and identical MDS coordinates [nodes 5 and 6; 7 and 8; 10 and 11; 12 and $13 ; 14$ and 15 - all are shown slightly displaced from their coordinate positions so that they do not overlap one another in Fig. 6(c)]. These species are the same pairs that were noted to be exactly regularly equivalent above. Outside of these species with identical ties in the network, the additive similarity measure did not uncover any nodes with similar network ties or position within the network, as the REGE coefficients did. The additive similarity measure grouped taxa independent of the direction of the ties - a major drawback. In addition, the top predators in the web M. nepenthicola (1) and T. klossi (3) are joined only after the fourth agglomeration step (Fig. 7), although they both clearly feed on aquatic insects living in the pitcher plants (Fig. 3). They feed on the same kinds of insects, but not the exact same species of insects, so they receive a low similarity in the additive similarity measure. But they do feed on the same trophic role class of insects (green), as can be seen in the MDS plot [Fig. 6(a)]. Also, (1) and (3) are joined at the same step as the bacteria and protozoa (16), yet these insects are the predators on the aquatic insect larvae group (4-9 and 12,13), whereas bacteria and protozoa are the prey of the aquatic larvae. Again, the direction of the ties is ignored by the additive similarity measure, which results in the same level of similarity for these species that occupy very different trophic roles.

Thus, the additive similarity measure appeared to do a poor job at uncovering trophic roles of insects in the pitcher plant food web, except in the cases where species had exactly the same predators and exactly the same prey. In contrast, REGE was far superior in finding similarity among species' trophic roles, even if the species consumed different prey and were consumed by different predators.

\subsection{ST. MARKS, FLORIDA SEAGRASS CARBON FLOW WEB}

We now turn to another example, using these same procedures to illustrate the method to aggregate trophically similar taxa in a complex food web. The 51 compartments in the food web collected by Baird et al. (1998) were analysed via the REGE algorithm, generating a matrix of coefficients (available from the authors and on-line at http://drjoe.biology.ecu.edu/regefoodweb). Appendix B lists the names and numerical identification codes for the compartments that are discussed here; see Christian \& Luczkovich (1999) and Luczkovich et al. (in press) for the complete species list for each compartment. The REGE coefficients were submitted to hierarchical clustering and $R^{2}$ values were computed for each partition. For display purposes we selected a partition with ten clusters, which achieved a much better $R^{2}$ than partitions with fewer clusters and explained nearly as much variance as partitions with more clusters (see scree plot, Fig. 8).

We used colors to identify the ten isotrophic classes of consumers in the MDS plot [Fig. 9(a)]: the lime green group, which was the main producer, the seagrass Halodule wrightii (2); the dark green group, which included other benthic producers [epiphytic algae (3) benthic algae (5)] and benthic bacteria (12); the cyan group, which included phytoplankton (1) and bacterio-plankton (6); the white group, which included zooplankton (8); the yellow group, which included various benthic invertebrates (nodes 7 , $9,13,15,17,19,21,22,25,27,28)$, some fishes

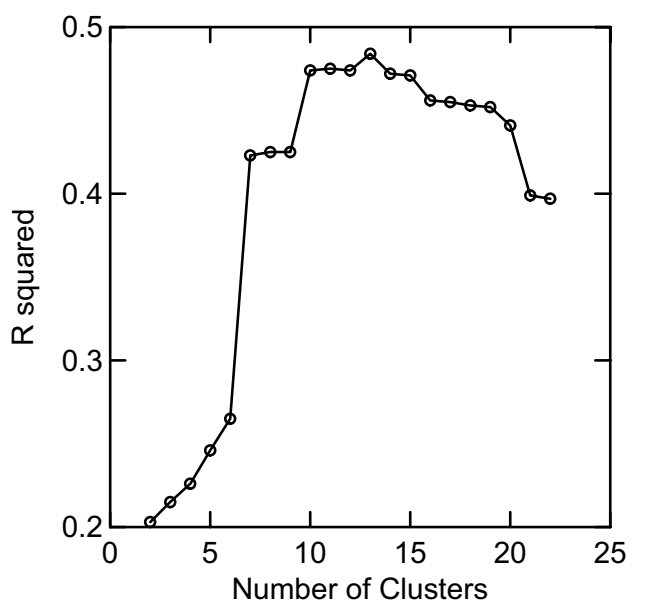

FIG. 8. A scree plot of the $R^{2}$ as it varies with number of clusters from the average linkage cluster analysis using the REGE coefficients derived from feeding relations and carbon-flow values of the St. Marks National Wildlife Refuge Seagrass Ecosystem Food Web 
(31) and suspended POC (50); the blue group, which included the remaining benthic macroinvertebrates (nodes 10, 11, 16, 18, 20, 23, 24, 26, 29,30 ) and most fishes (nodes 32, 33, 35, 37-41); the purple group, which included the remaining fishes (nodes 34,36,42); the magenta group, which included herbivorous ducks (43); the red group, which included carnivorous birds (nodes 44-48); and the brown group, which included meiofauna (14), and non-living groups [dissolved organic carbon (DOC) (49) and sediment particulate organic carbon (POC) (51)]. Most producers (nodes 1-5) and non-living compartments (nodes 49,51 ) appear on the right side of the MDS plot, the intermediate consumers (nodes 7 42 ) in the center of the plot, and the carnivorous birds (nodes 44-48) at the lower left side of the plot. Herbivorous ducks (43) are at the far left, implying that they are a top predator (and they are not preyed upon by other compartments in this web), although they are separated from other top carnivore birds. Thus, the plot produces a food web that shows the approximate trophic positions of all the taxa, moving from low trophic positions on the right to high trophic positions on the left.

Pairs of compartments that are close together in the MDS plot have similar trophic roles (e.g. they share high REGE coefficients), which implies that they have similar predators as well as similar prey. For example, the pelagic and benthic food webs can be separated: planktonic compartments $(1,6,8)$ occur in the upper right and center portion of plot, and benthic species at the lower right portion of the plot $(3,5,12,14$, 51). Because some consumer species are inter- mediate in their trophic roles (feed on benthic and planktonic species and are omnivores), they appear in the center of the MDS plot. The bacterial consumer compartments [bacterioplankton (6) and benthic bacteria (12)] are grouped with the producers in the MDS, in part because they feed upon sediment POC and are fed upon by species that are otherwise detritivores and herbivores. In this food web, the carnivorous and herbivorous birds are not linked to the detrital compartment (51), but all other consumers are, because the top carnivores' carbon has been assumed to migrate out of the system (Baird et al, 1998; Christian \& Luczkovich, 1999), consistent with the migratory nature of these birds. This similar migration pattern and absence of connections to the sediment POC (51) caused all the birds to occur together on the left side of the MDS plot.

We collapsed the same-colored nodes and generated an image graph [Fig. 9(b)] that shows the structure of the food web, while retaining the relationships among classes that define the isotrophic groupings. The isotrophic classes we have identified are clearly associated with specific trophic roles in the St. Marks food web. The lime and dark green groups included benthic producers. The cyan group included planktonic producers and the white group included planktonic consumers (zooplankton). The yellow group included benthic invertebrates and fishes that consumed benthic and planktonic producers, benthic bacteria, and other consumers (white, blue and purple groups). The yellow group consumed members of their own group (shown as a self-loop in the figure); they are in

FIG. 6. (a) A display of the two-dimensional non-metric multi-dimensional scaling of the REGE coefficients from the Malaysian pitcher plant insect food web using the network software Pajek (version 0.83 ). Arrows trace the path of energy in the food web. Colors were used to indentify class membership based on the $R^{2}$ regression analysis (see text, Figs 4 and 5). Taxa which have identical links and thus REGE coefficients of 1.0 (5 and 6, 7 and 8,10 and 11,12 and 13,14 and 15) have been plotted with a slight offset so that they do not obscure one another. (b) An image graph of the Malaysian pitcher plant insect food web based on the regular equivalence relations shown in (a). (c) The food web network displayed as a twodimensional non-metric multi-dimensional scaling of the additive similarity coefficients of Yodzis \& Winemiller (1999).

FIG. 9. (a) A non-metric-multi-dimensional scaling of all nodes in the St. Marks seagrass ecosystem carbon-flow food web using REGE-derived isotrophic classes from the cluster analysis, and (b) the image graph of the reduced complexity network of the same food web. Colors were used to indentify class membership based on the $R^{2}$ regression analysis (see text and Fig. 8). In the image graph, the codes for blue class $\{A\}$ are $\{4,10,11,16,18,20,23,24,26,29,30,32,33,35,37,38,39$, $40,41\}$ and yellow class $\{$ B $\}$ are $\{7,9,13,15,17,19,21,22,25,27,28,31,50\}$. See Appendix B for a complete list of compartment identification code names. 

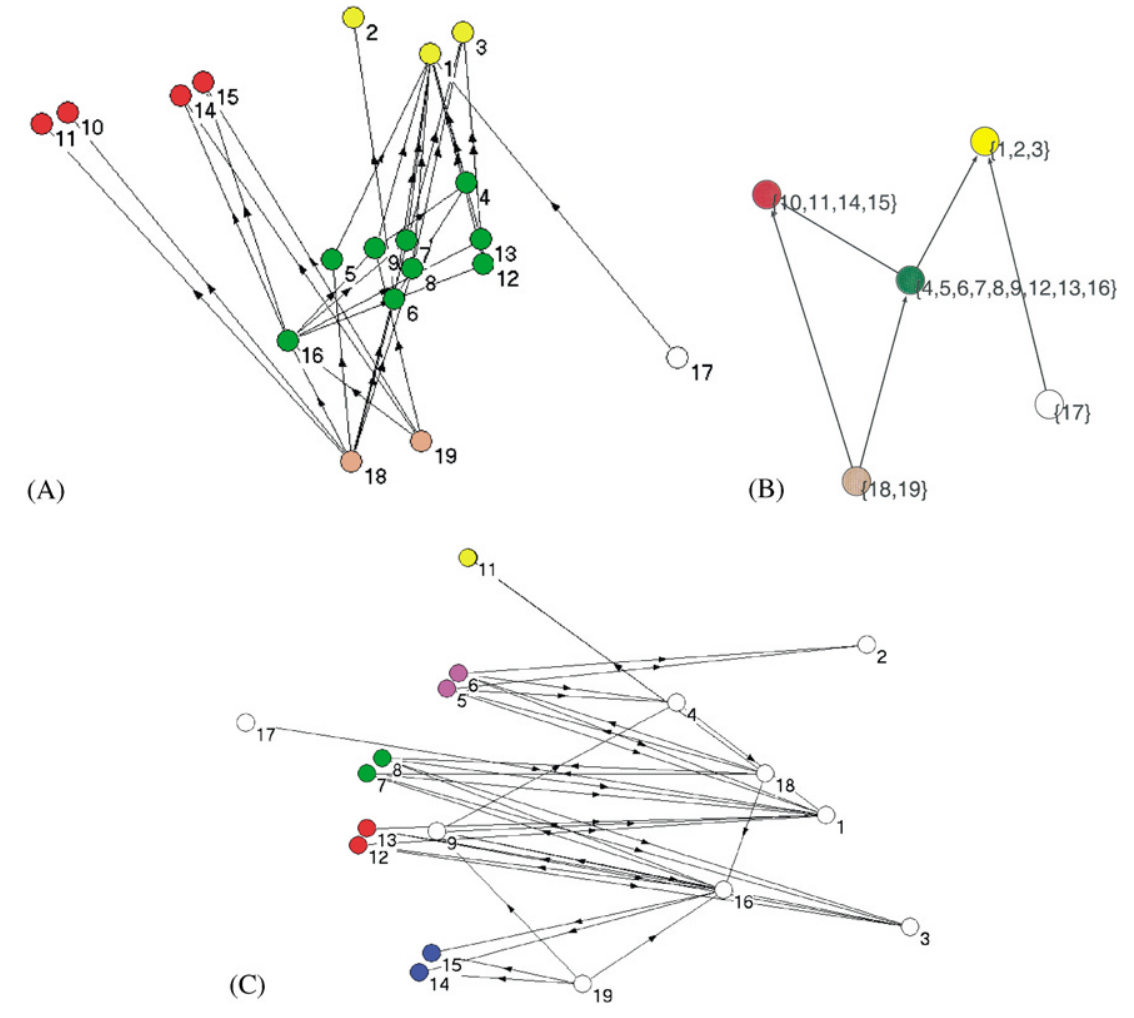

FIG. 6.
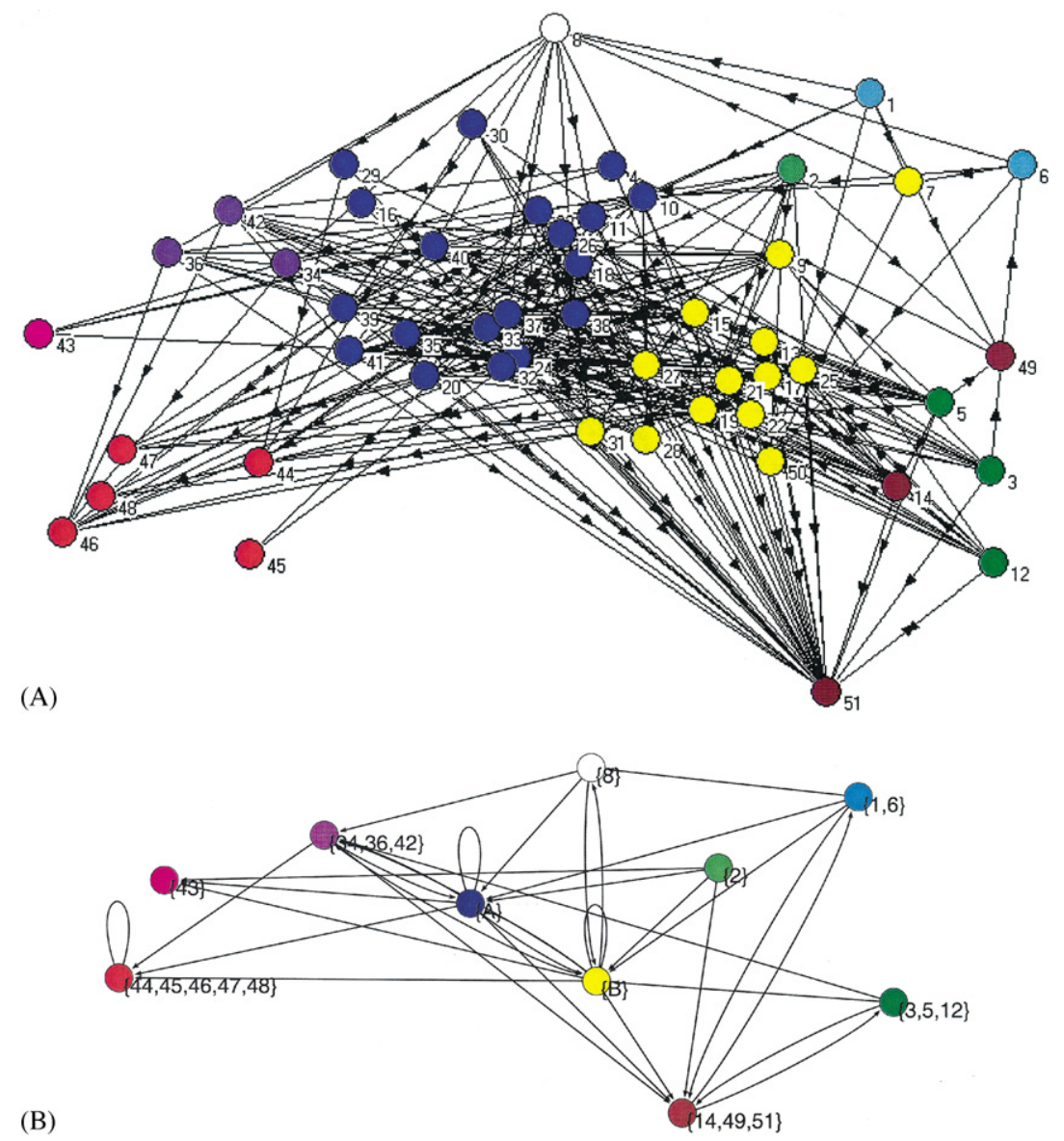

FIG. 9.

(B) 

turn preyed upon by the zooplankton (white), fishes and invertebrates in the blue and purple groups, and birds in the magenta and red groups. Yellow group members thus take part in many loops and cycles with blue, purple and white. In contrast, the blue group members (mostly fish and invertebrates) take part in a cycle only with yellow and purple members. Blue group members feed upon planktonic producers, but only one class of benthic producers (Halodule, lime green group, and not the benthic algae, epiphytes or benthic bacteria, dark green group). Blue group members feed upon members of their own class, as do yellow group members, but they do not have members that are consumed by zooplankton (white), unlike yellow group members. Members of the blue group are consumed by members of the purple, magenta and red groups and are thus somewhat similar to the yellow group members. The purple group members are fishes that consumed the dark green group of benthic producers, but not planktonic producers, nor the seagrass benthic producers (lime green); they are different from the yellow and blue groups in this way. In addition, they do not have any feeding within their own group. The purple group also consumes white, yellow and blue group members. They are consumed only by the carnivorous birds (red group), but not the herbivorous ducks (magenta). The herbivorous ducks (magenta) are in their own singleton class; no other bird group feeds on producers (but these ducks also take some animal prey from the yellow and blue groups, so they are not strictly speaking herbivorous). The red group (predatory birds) feeds from the yellow, blue and purple classes and has one member that consumes another (raptors consume gulls). All compartments except for the birds (red and magenta) are consumed by the brown group, which includes detritus [sediment POC (51)].

\section{Discussion}

A major goal in ecology is to develop a comprehensive model of the ecological niche or role played by a species in a community or ecosystem. One way that the ecologists have worked toward this goal is through the analysis of trophic relations in food webs. Concepts such as of trophospecies, trophic role, trophic group, trophic position, trophic level, guild and related concepts pertaining to food webs have been developed, all of which are attempts to reduce trophic complexity by identifying classes of "isotrophic" species that occupy analogous positions in the food web. Our contributions to this endeavor are at two levels. First, we contribute to the goal of comprehending trophic roles in ecosystems by providing a formal mathematical basis, expressing trophic role in terms of equivalence relations and graph homomorphisms. In this respect, we extend the work of Yodzis \& Winemiller (1999), who developed measures and algorithms for detecting structure in food webs, by locating their work within a framework of fundamental mathematical notions of equivalence and isomorphism. In addition, we link their work to research in the social sciences (Lorrain \& White, 1971) and computer sciences (Starke 1972), among other fields.

Second, we introduce a specific model of food web structure - regular equivalence — which we believe captures the notion of trophic role better than other formal models, such as those offered by Yodzis \& Winemiller (1999) and Hirata \& Ulanowicz (1985). The recursive nature of the definition might suggest that computation of the model would be difficult, but in fact very efficient algorithms exist, such as REGE, which provide a measure of the degree of equivalence among all pairs of species, and these algorithms are widely available (Borgatti et al., 1999). We have illustrated our approach here using two food webs from the literature, namely the Malaysian pitcher plant insect topological food web and the St. Marks seagrass ecosystem carbon-flow dynamic food web. We have also applied the approach to rocky intertidal, desert, and rainforest food webs (Johnson et al. 2000).

The regular equivalence model we introduce here contrasts with structural equivalence, in which nodes are equivalent to the extent they have the same kind of ties to the same individuals (rather than classes of individuals). We show that the additive trophic similarity measure recently developed by Yodzis \& Winemiller (1999) is a measure of structural equivalence. Alternative measures of structural 
equivalence, based on euclidean distances and correlations, as well alternative algorithms, are well known in the social sciences and are available in standard software packages. Structural equivalence is ideally suited for certain analytical tasks. Structurally equivalent species are literally are structurally indistinguishable, tied to the same others in the same ways. Consequently, for all structural ecological processes - by which we mean processes whose outcomes are principally determined by the structure of the trophic network - we can expect structurally equivalent species to have similar outcomes, or common fate (Homans, 1950). Thus, in trophic impact studies, anything that happens to a species of predator or prey is expected to happen to all other species in the same structural equivalence class, because they have exactly the same prey or predators. In this respect, structural equivalence may have an advantage over maximal regular equivalence, as it is unclear if or under what conditions the common fate hypothesis can be applied to regular equivalence classes.

The disadvantage of structural equivalence is that it cannot recognize the structural similarity of species that share no predators or prey. Consequently, species in clearly analogous positions, which are functionally equivalent, are seen as completely dissimilar. For example, in the Malaysian insect food web, food sources that are similar (organic debris and recently drowned insects) were not regarded as similar by the structural method of Yodzis \& Winemiller (1999), yet they clearly are similar in that they consume nothing and are eaten by similar species. Regular equivalence solves this problem of not detecting similarity in trophic role when species share no or very few prey or predators.

Regular equivalence can be used to find species playing similar roles in spatially and temporally separate food webs in which species do not consume the exact same prey, possibly opening the door for new kinds of comparative research. For example, in Jackson et al. (2001), the authors proposed that humans have altered coastal ecosystems by fishing and hunting activities that have caused long-lasting food web changes. In the Pacific kelp forest food web, when sea otters were extirpated, there was a decades-long lag response in the overgrazing effect of sea urchins, their primary prey. The lag was due to the fact that other unexploited species such as abalones and spiny lobsters "... of a similar trophic level assumed the ecological roles of overfished species... (emphasis ours) (p. 629)". Our regular equivalence analysis would allow a quantitative assessment of the similarity of these species' trophic roles, even when they did not prey on the same species and were preyed upon by different species. Thus, we can create a simple model of trophically redundant relationships when they occur in the same food web at different points in time, or even in spatially separate food webs. While structural equivalence may also be used in such an example, it would be better for studying direct trophic impacts and modeling direct competition between species, whereas regular equivalence could be used to model the potential for species to overlap in trophic role, should their feeding habits shift as suggested by Jackson et al. (2001).

The approach we have outlined has some additional benefits in quantifying trophic roles and displaying reduced complexity food webs. Like the approach of Yodzis \& Winemiller (1999), we have proposed a method that will find similarity among species based on their relationships with predators and prey. This then forms a new basis for aggregating species.

The problem of how to aggregate nodes in a network of interactions so as to eliminate detail while retaining structure is universal in system modeling. In fact, this issue has been formally approached previously in ecological network analysis. A method for network aggregation was proposed by Hirata \& Ulanowicz (1985), in which a 17-compartment carbon-flow network of a tidal marsh creek was aggregated into a seven-compartment system by minimizing the loss of mutual information that occurred during a sequence of aggregation steps. Their graph of the seven-compartment ecosystem flow diagram was essentially an image graph, but the aggregations were based on minimizing the decrease in network mutual information, an empirical statistic they computed, which is based on network redundancy measures. Although the general approach is similar to ours, Hirata \& 
Ulanowicz's (1985) node aggregation approach is quite different in mathematical detail from ours, and we plan to compare them systematically in future work.

Although we have emphasized the differences between our maximal regular equivalence approach and the structural equivalence approaches of Yodzis \& Winemiller (1999), it is important to remember that there is a fundamental commonality as well. As noted earlier, structural equivalence is a member of the regular equivalence family, and any species that are structurally equivalent are necessarily regularly equivalent, as we illustrated in the Malaysian pitcher plant food web example. In that example, four pairs of nodes with the exact same predator and prey relationships were found to be structurally equivalent, and they were also exactly regularly equivalent. The similarity of trophic relations between any two nodes could be determined using the structural methods alone when their nearest-neighbor links were considered; but when similarity among nodes was estimated while considering links that were more than a one link away from the nodes, such similarity could be estimated using REGE coefficients. If the equivalence approach provides lenses with which to view food webs, the structural equivalence provides finer resolution at the expense of the big picture, while regular equivalence provides the big picture at the expense of only a few details.

A key area for future research concerns the significance of isotrophic classes — whether defined by structural equivalence or regular equivalence - for the species that comprise them and for the webs as a whole. Yodzis \& Winemiller (1999) evaluate the relative merits of two different measures of trophic similarity by using cophenetic correlations. But a cophenetic correlation only measures the extent to which a given similarity matrix can be nicely represented by a dendrogram (i.e. a hierarchical clustering procedure). It is not an external criterion of validity. What is needed is empirical research relating membership in isotrophic classes to outcome criteria, such as similar reactions to environmental changes, similar effects on prey species, similar responses to the removal or addition of predator species, and so on. Only then can we really determine whether one definition of trophic grouping is more useful than other (and whether any kind of trophic grouping is useful at all).

This work was supported by a Biocomplexity Incubation grant from the National Science Foundation (SES0083508). The East Carolina University Division of Research and Graduate Studies supported the authors in the preparation of this paper and provided publication costs. We thank P. Stiling for taxonomic assistance and verification of the insect food web data. B. Patten is acknowledged for providing helpful criticisms. We also thank R. Ulanowicz and $\mathrm{R}$. Bernard for reviewing the manuscript.

\section{REFERENCES}

Adams, S. M., Kimmel, B. L. \& Ploskey, G. R. (1983). Sources of organic matter for reservoir fish production: a trophic-dynamics analysis. Can. J. Fish. Aquat. Sci. 40, 1480-1495.

Baird, D., Luczkovich, J. J. \& Christian, R. R. (1998). Assessment of spatial and temporal variability in ecosystem attributes of the St. Marks National Wildlife Refuge, Apalachee Bay, Florida. Estuarine Coastal Shelf Sci. 47, 329-349, doi:10.1006/ecss.1998.0360.

Batagelu, V. \& Mrvar, A. (1999). Pajek, version 0.83, available on-line at http://vlado.fmf.uni-lj.si/pub/networks/pajek.

Beaver, R. A. (1985). Geographical variation in food web structure in Nepenthes pitcher plants. Ecol. Entomol. 10, 241-248.

Borgatti, S. P. \& Everett, M. G. (1989). The class of all regular equivalences: algebraic structure and computation. Soc. Networks 11, 65-88.

Borgatti, S. P. \& Everett, M. G. (1993). Two algorithms for computing regular equivalence. Soc. Networks 16, 43-55.

Borgatti, S. P., Everett, M. G. \& Shirey, P. (1990). LS sets, lambda sets, and other cohesive subsets. Soc. Networks 12, 337-357.

Borgatti, S. P., Everett, M. G. \& Freeman, L. (1999). UCINET $V$. Software for Social Network Analysis. Harvard, MA: Analytical Technologies.

Boyd, J. P. (1969). The algebra of group kinship. J. Math. Psychol. 6, 139-167.

Christian, R. R. \& Luczkovich, J. J. (1999). Organizing and understanding a winter's seagrass foodweb network through effective trophic levels. Ecol. Model. 117, 99-124.

Cohen, J. E. \& Newman, C. M. (1985). A stochastic theory of community food webs. I. Models and aggregated data. Proc. R. Soc. London Biol. Sci. 224, 421-448.

Cousins, S. H. (1985). Ecologists build pyramids again. New Sci. 106, 50-54.

Cousins, S. H. (1987). The decline of the trophic level concept. Trends Ecol. Evol. 2, 312-316.

Elton, C. S. (1927). Animal Ecology. London: Sidgwick and Jackson. 
Everett, M. G. \& Borgatti, S. P. (1991). Role colouring a graph. Math. Soc. Sci. 21, 183-188.

Everett, M. G. \& Borgatti, S. P. (1994). Regular equivalence: General theory. J. Math. Soc. 19, 29-52.

Everett, M. G. \& Borgatti, S. P. (1996). Exact colorations of graphs and digraphs. Soc. Networks 18, 319-331.

Goldwasser, L. \& Roughgarden, J. (1993). Construction and analysis of a large Caribbean food web. Ecology 74, 1216-1233.

Goodenough, W. H. (1969). Rethinking "status" and "role": toward a general model of the cultural organization of social relationships. In Cognitive Anthropology (Tyler, S. A., ed.), pp. 311-330. New York: Holt, Rinehart, and Winston.

Hirata, H. \& Ulanowicz, R. E. (1985). Information theoretical analysis of the aggregation and hierarchical structure of ecological networks. J. theor. Biol. 116, 321-341.

Homans, G. C. (1950). The Human Group. New York: Harcourt, Brace, and World.

Homans, G. C. (1961). Social Behavior: Its Elementary Forms. New York: Harcourt, Brace, and World.

Hutchinson, G. E. (1958). Concluding remarks. Cold Spring Harbor Symp. Quant. Biol. 22, 415-427.

JACCARD, P. (1900). Contribution au problame de l'immigration post-glaciaire de la flore alpine. Bull. Soc. Vaudoise Sci. Nat. 36, 87-130.

JACKSON, J. B. C., Kirby, M. X., Berger, W. H., BJorndal, K. J., Botsford, L. W., Bourque, B. J., Bradbury, R. H., Cooke, R., Erlandson, J., Estes, J. A., Hughes, T. P., Kidwell, S., Lange, C. B., Lenihan, H. S., Pandolfi, J. M., Peterson, C. H., Steneck, R. S., Tegner, M. J. \& WARNER, R. H. (2001). Historical overfishing and the recent collapse of coastal ecosystems. Science 293, 629-638.

Johnson, J. C., Borgatti, S. P., Luczkovich, J. J. \& Everett, M. G. (2001). Network role analysis in the study of food webs: an application of regular role coloration. J. Soc. Struct. 2, on-line: http://www.heinz. cmu.edu/project/INSNA/joss/index 1.html.

JoHnson, S. C. (1967). Hierarchical clustering schemes. Psychometrika 32, 241-253.

LindEMAN, R. L. (1942). The trophic-dynamic aspect of ecology. Ecology 23, 399-418.

Lorrain, F. \& White, H. C. (1971). Structural equivalence of individuals in networks. J. Math. Soc. 1, 49-80.

Luczkovich, J. J., Ward, G. P., Johnson, J. C., Christian, R. R., Baird, D., Neckles, H. \& Rizzo, B. Determining the trophic guilds of fishes and macroinvertebrates in a seagrass food web. Estuaries, in press.

MayHew, B. (1980). Structuralism vs. individualism. Part 1: shadow boxing in the dark. Soc. Forces 59, 335-375.

Merton, R. K. (1959). Social Theory and Social Structure. New York: Free Press.

NADEL, S. F. (1957). The Theory of Social Structure. New York: Free Press.

Perrson, L., Bengtsson, J., Menge, B. A. \& Power, M. E. (1996). Productivity and consumer regulation concepts, patterns, and mechanisms. In: Food Webs: Integration of Patterns and Dynamics. (Polis, G. \& Winemiller, K. O., eds), pp. 396-434. Chapman \& Hall. New York, NY, U.S.A.
Root, R. B. (1967). The niche exploitation pattern of the blue-gray gnatcatcher. Ecol. Monogr. 37, 317-350.

Starke, P. H. (1972). Abstract Automata. Amsterdam: North Holland Publishing Company, Elsevier.

Stiling, P. (2002). Ecology: Theories and Applications. Upper Saddle River, NJ: Prentice-Hall.

Ulanowicz, R. E. (1986). Growth and Development: Ecosystems Phenomenology. New York: Springer-Verlag.

White, H. C. (1963). An Anatomy of Kinship. Englewood Cliffs, NJ: Prentice-Hall.

White, H. C. \& Reitz, K. P. (1983). Graph and semigroup homomorphisms on networks of relations. Soc. Networks 5, 193-235.

YoDZIS, P. (1988). The indeterminacy of ecological interactions as perceived through perturbation experiments. Ecology 69, 508-515.

Yodzis, P. \& Winemiller, K. O. (1999). In search of operational trophospecies in a tropical aquatic food web. Oikos 87, 327-340.

\section{APPENDIX A}

A list of the species of insects and compartments in the Malaysian pitcher plant topological food web (Beaver, 1985), taken from Stiling (2002).

(1) Misumenops nepenthicola, (2) Encyrtid (near Trachinaephagus), (3) Toxorhynchies klossi, (4) Lestodiplosis syingopais, (5) Megaselia nepenthina, (6) Endonepenthinia schuitemakeri, (7) Triperoides tenax, (8) Triperoides bambusa, (9) Dasyhelea nepenthicola, (10) Nepenthosyrphus sp., (11) Pierretia urceola, (12) Culex curtipalpis, (13) Culex lucaris, (14) Anotidae sp. 1, (15) Anotidae sp. 2, (16) bacteria and protozoa, (17) live insects, (18) recently drowned insects and (19) older organic debris.

\section{APPENDIX B}

A list of the identification codes and compartment names for the St. Marks seagrass carbonflow food web taken from Baird et al. (1998). See Christian \& Luczkovich (1999) for a complete species list within each compartment.

(1) Phytoplankton, (2) Halodule wrighti, (3) micro-epiphytes, (4) macro-epiphytes, (5) benthic algae, (6) bacterio-plankton, (7) microprotozoa, (8) zooplankton, (9) epiphyte-grazing amphipods, (10) suspension-feed molluscs, (11) suspension-feed polychaetes, (12) benthic bacteria, 
(13) micro-fauna, (14) meiofauna, (15) depositfeeding amphipods, (16) detritus-feeding crustaceans, (17) hermit crab, (18) spider crab, (19) omnivorous crabs, (20) blue crab, (21) isopod, (22) brittle stars, (23) herbivorous shrimp, (24) predatory shrimp, (25) deposit-feeding gastropod, (26) deposit-feeding polychaetes, (27) predatory polychaetes, (28) predatory gastropod, (29) epiphyte-grazing gastropod, (30) other gastropods, (31) catfish and stingrays, (32) tonguefish, (33) gulf flounder and needle fish,
(34) southern hake and sea robins, (35) atlantic silverside and bay anchovies, (36) gobies and blennies, (37) pinfish, (38) spot, (39) pipefish and seahorses, (40) sheepshead minnow, (41) red drum, (42) killifish, (43) herbivorous ducks, (44) benthos-eating birds, (45) fish-eating birds, (46) fish and crustacean eating birds, (47) gulls, (48) raptors, (49) dissolved organic carbon (DOC), (50) suspended particulate organic carbon, (51) sediment POC. 